\title{
Robust Distributed Model Predictive Load Frequency Control of Interconnected Power System
}

\author{
Xiangjie Liu, ${ }^{1}$ Huiyun Nong, ${ }^{1}$ Ke Xi, ${ }^{1}$ and Xiuming Yao ${ }^{2}$ \\ ${ }^{1}$ State Key Laboratory of Alternate Electrical Power System with Renewable Energy Sources, North China Electric Power University, \\ Beijing 102206, China \\ ${ }^{2}$ Department of Automation, North China Electric Power University, Baoding 071003, China
}

Correspondence should be addressed to Xiangjie Liu; liuxj@ncepu.edu.cn

Received 10 August 2013; Accepted 20 September 2013

Academic Editor: Zhiguang Feng

Copyright (C) 2013 Xiangjie Liu et al. This is an open access article distributed under the Creative Commons Attribution License, which permits unrestricted use, distribution, and reproduction in any medium, provided the original work is properly cited.

\begin{abstract}
Considering the load frequency control (LFC) of large-scale power system, a robust distributed model predictive control (RDMPC) is presented. The system uncertainty according to power system parameter variation alone with the generation rate constraints (GRC) is included in the synthesis procedure. The entire power system is composed of several control areas, and the problem is formulated as convex optimization problem with linear matrix inequalities (LMI) that can be solved efficiently. It minimizes an upper bound on a robust performance objective for each subsystem. Simulation results show good dynamic response and robustness in the presence of power system dynamic uncertainties.
\end{abstract}

\section{Introduction}

The load frequency control (LFC) has long been a much concerned research interest for power system engineers over the past forty years [1]. In modern power system, undesirable frequency and scheduled tie-line power changes in multiarea power system are a direct result of the imbalance between generated power and system demand plus associated system losses. The main objectives of the LFC are to keep the system frequency at the scheduled value and regulate the generator units to make the area control error tend to zero under the continuous adjustment of active power, so that the generation of the entire system and the load power well match.

In a practical power system, there exist different kinds of uncertainties, such as changes in parameter. And each control area contains various disturbances due to increased complexity, system modeling errors, and changing power system structure. Thus the robustness must be taken into theoretical consideration in the LFC design procedure to promise high power quality. A fixed controller based on classical theory is not very suitable for the LFC problem. It is necessary that a flexible controller should be developed [2-4]. Robust LFC was early designed based on the Riccati equation approach [5], which is simple and effective and can ensure the overall system to be asymptotically stable for all admissible uncertainties. Motivated by the large uncertainty in dynamic models of power system components and their interconnections, paper [6] proposes a physically motivated passivity objective as a means to achieve effective closed-loop control. Recently, robust LFC can be realized using linear matrix inequalities [7], fuzzy logic [8], neural networks [9], and genetic algorithms [10].

Model predictive control (MPC) has been an attracting method for power system LFC, which can perform an optimization procedure to calculate optimal control actions within the realistic power system constrains. In LFC research, there is the practical limit on the rate of change in the generating power, called the generation rate constraints (GRC), which can result in the LFC to be a constraint optimal problem. Traditional MPC is unable to explicitly incorporate plant uncertainty. Thus, robust MPC has been well developed recently $[11,12]$.

Most existing MPCs assume that all subsystems are identical, which is not the case of actual power systems. Subsequently, a number of decentralized/distributed load frequency controllers were developed to eliminate the above 
drawback. In [13], the distributed model predictive control (DMPC) is used in LFC, which offers an effective means of achieving the desired controller coordination and performance improvements. A decentralized MPC framework for multiarea power system has been presented in [14]. Accordingly, the robustness of DMPC strategies to model errors has been identified as a key factor for the successful application of DMPC [15].

In this paper, a robust distributed MPC (RDMPC) strategy for load frequency control in interconnected power system is presented. The entire power system is composed of several subareas and the problem is formulated as convex optimization problem with linear matrix inequalities (LMI) that can be solved efficiently using the algorithm. The method shows good dynamic response and robustness in the presence of power system model dynamic uncertainties.

\section{Mathematical Model of Power System}

The interconnected power system consists of at least two control areas connected by tie lines. Usually the subsystem contains thermal power system, hydro power system, nuclear power system, and renewable power system, in which thermal power system and hydro power system generally participate in load frequency control. Figures 1 and 2 show, respectively, the structures of thermal power plant and hydro power plant in power system LFC. The original model has been given in [16]. Comparing to [16], the model in this article contains the reheater part, which is quite common in modern thermal power plant. Each control area has its own controller. The variables and parameters are given in Table 1.

When load change happens in one area, all the interconnected areas will be affected, and the controllers act to adjust the frequency deviation and tie-line active power to return to steady state. The LFC using RDMPC will be applied to the whole control areas.

The time-varying linearized mathematical model of thermal and hydro plant used in interconnected power system can be described as

$$
\begin{aligned}
\dot{\mathbf{x}}_{i}(t)= & \mathbf{A}_{i i}(t) \mathbf{x}_{i}(t) \\
& +\sum_{j} \mathbf{A}_{i j}(t) \mathbf{x}_{j}(t)+\mathbf{B}_{i i}(t) \mathbf{u}_{i}(t)+\mathbf{F}_{i i}(t) \mathbf{d}_{i}(t),
\end{aligned}
$$

where $i$ represents the control area; $\mathbf{x}_{i} \in \mathbb{R}^{n_{i}}, \mathbf{u}_{i} \in \mathbb{R}^{m_{i}}$, and $\mathbf{d}_{i} \in \mathbb{R}^{z_{i}}$ represent the state, input, and disturbance vector in the $i$ 's subsystem, respectively. $\mathbf{x}_{j} \in \mathbb{R}^{n_{j}}$ is a state vector of the neighbor system.

Define the area control error (ACE) to be

$$
\mathrm{ACE}_{i}=\mathbf{y}_{i}(t)=\mathbf{C}_{i i} \mathbf{x}_{i}(t)=B_{i} \Delta f_{i}(t)+\Delta P_{\text {tiei }}(t),
$$

where $\mathbf{y}_{i} \in \mathbb{R}^{v_{i}}$ represents system output signal. Matrices in (1) and (2) have dimensions

$$
\begin{gathered}
\mathbf{A}_{i i} \in \mathbb{R}^{n_{i} \times n_{i}}, \quad \mathbf{A}_{i j} \in \mathbb{R}^{n_{i} \times n_{j}}, \quad \mathbf{B}_{i i} \in \mathbb{R}^{n_{i} \times m_{i}}, \\
\mathbf{F}_{i i} \in \mathbb{R}^{n_{i} \times z_{i}}, \quad \mathbf{C}_{i i} \in \mathbb{R}^{v_{i} \times n_{i}} .
\end{gathered}
$$

In Figure 1, the state variable in the thermal power system is

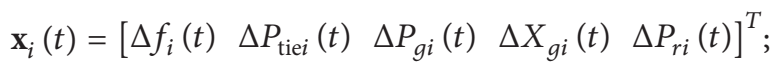

while the state variable in the hydro power system is

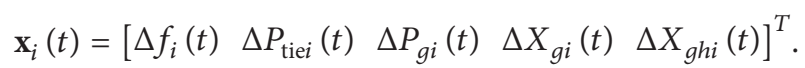

The control signal and disturbance in both the thermal power system and the hydro power system are as follows:

$$
\mathbf{u}_{i}(t)=\Delta P_{c i}(t), \quad \mathbf{d}_{i}(t)=\Delta P_{d i}(t) .
$$

The state, control, and disturbance matrix in thermal power system are

$$
\begin{gathered}
\mathbf{A}_{i i}=\left[\begin{array}{ccccc}
-\frac{1}{T_{p i}} & -\frac{K_{p i}}{T_{p i}} & \frac{K_{p i}}{T_{p i}} & 0 & 0 \\
\sum_{j \neq i} T_{s i j} & 0 & 0 & 0 & 0 \\
0 & 0 & -\frac{1}{T_{T i}} & 0 & \frac{1}{T_{T i}} \\
-\frac{1}{T_{G i} R_{i}} & 0 & 0 & -\frac{1}{T_{G i}} & 0 \\
-\frac{K_{r i}}{R_{i} T_{G i}} & 0 & 0 & \frac{1}{T_{r i}}-\frac{K_{r i}}{T_{G i}} & -\frac{1}{T_{r i}}
\end{array}\right], \\
\mathbf{B}_{i i}=\left[\begin{array}{c}
0 \\
0 \\
0 \\
\frac{1}{T_{G i}} \\
0
\end{array}\right], \\
\mathbf{F}_{i i}=\left[\begin{array}{c}
-\frac{K_{p i}}{T_{p i}} \\
0 \\
0 \\
0 \\
0
\end{array}\right]
\end{gathered}
$$

while the state, control, and disturbance matrix in hydro power system are

$$
\begin{gathered}
\mathbf{A}_{i i}=\left[\begin{array}{ccccc}
-\frac{1}{T_{P i}} & -\frac{K_{P i}}{T_{P i}} & \frac{K_{P i}}{T_{P i}} & 0 & 0 \\
\sum_{j \neq i} T_{s i j} & 0 & 0 & 0 & 0 \\
2 \alpha & 0 & -\frac{2}{T_{W i}} & 2 \kappa & 2 \beta \\
-\alpha & 0 & 0 & -\frac{1}{T_{2 i}} & -\beta \\
-\frac{1}{T_{1 i} R_{i}} & 0 & 0 & 0 & -\frac{1}{T_{1 i}}
\end{array}\right], \\
\mathbf{B}_{i i}=\left[\begin{array}{c}
0 \\
0 \\
-2 R_{i} \beta \\
R_{i} \beta \\
\frac{1}{T_{1 i}}
\end{array}\right], \\
\mathbf{F}_{i i}=\left[\begin{array}{c}
K_{P i} \\
T_{P i} \\
0 \\
0 \\
0 \\
0
\end{array}\right],
\end{gathered}
$$




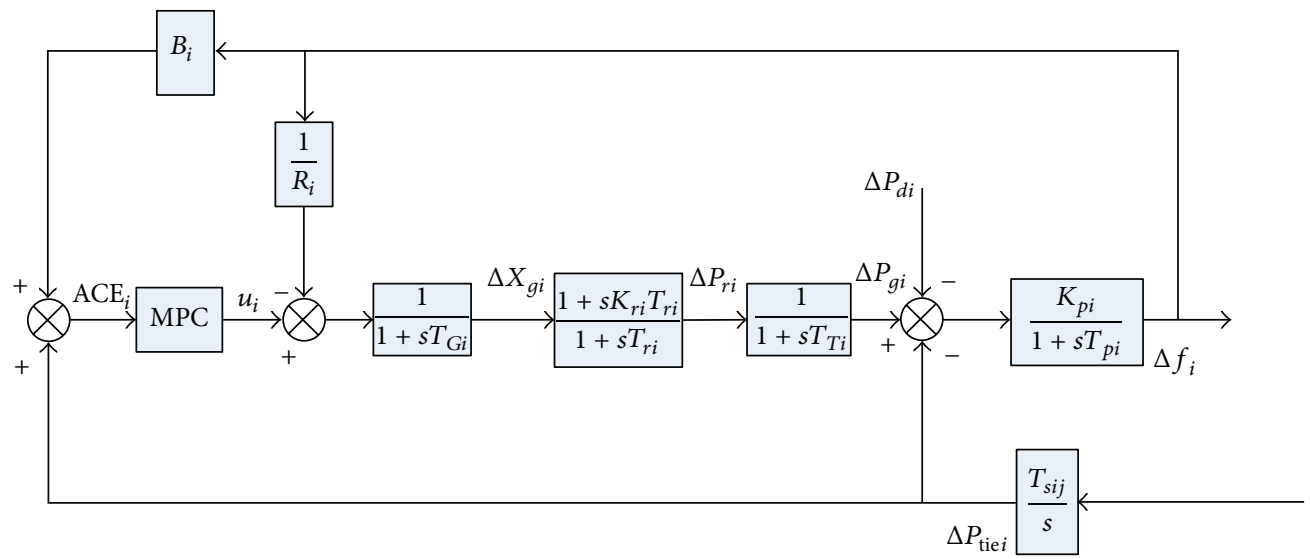

FIGURE 1: The block diagram of thermal power plant in area $i$.

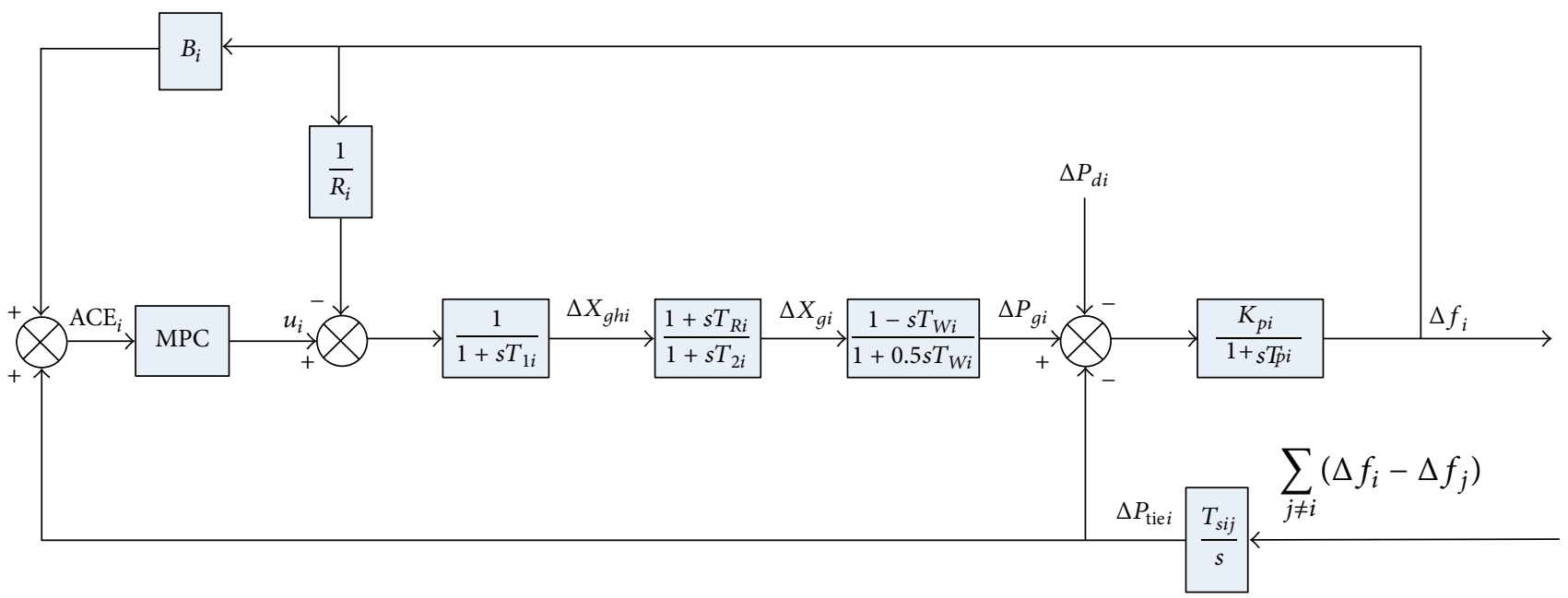

FIGURE 2: The block diagram of hydro power plant in area $i$.

where $\alpha=T_{R i} / T_{1 i} T_{2 i} R_{i}, \beta=\left(T_{R i}-T_{1 i}\right) / T_{1 i} T_{2 i}, \kappa=\left(T_{2 i}+\right.$ $\left.T_{W i}\right) / T_{2 i} T_{W i}$.

Here $A_{i j}$ have $5 \times 5$ dimensions. All their elements are equal to zero, except for the element at position $(1,2)$, which is equal to $-T_{s i j}$.

For the whole power system, the state-space equation is as follows:

$$
\begin{aligned}
\dot{\mathbf{x}}(t)=\mathbf{A}(t) x(t) & +\mathbf{B}(t) u(t)+\mathbf{F}(t) d(t), \\
\mathbf{y}(t) & =\mathbf{C}(t) x(t),
\end{aligned}
$$

where

$$
\begin{array}{cc}
\mathbf{x}(t)=\left[\begin{array}{c}
\mathbf{x}_{1}(t) \\
\mathbf{x}_{2}(t) \\
\vdots \\
\mathbf{x}_{M}(t)
\end{array}\right] & \mathbf{u}(t)=\left[\begin{array}{c}
\mathbf{u}_{1}(t) \\
\mathbf{u}_{2}(t) \\
\vdots \\
\mathbf{u}_{M}(t)
\end{array}\right], \\
\mathbf{d}(t)=\left[\begin{array}{c}
\mathbf{d}_{1}(t) \\
\mathbf{d}_{2}(t) \\
\vdots \\
\mathbf{d}_{M}(t)
\end{array}\right] \quad \mathbf{y}(t)=\left[\begin{array}{c}
\mathbf{y}_{1}(t) \\
\mathbf{y}_{2}(t) \\
\vdots \\
\mathbf{y}_{M}(t)
\end{array}\right] .
\end{array}
$$

This is a general continuous-time linear system with added disturbance. $M$ is the number of control areas of the interconnected power system. After using the zero-order hold $(\mathrm{ZOH})$ discretization method, each control area's distributed discrete-time linear model is expressed as follows:

$$
\begin{aligned}
x_{i}(k+1)= & \widetilde{A}_{i i}(k) x_{i}(k)+\widetilde{B}_{i i}(k) u_{i}(k) \\
& +\sum_{j \neq i}^{M}\left(\widetilde{A}_{i j}(k) x_{j}(k)+\widetilde{B}_{i j}(k) u_{j}(k)\right),
\end{aligned}
$$

$$
y_{i}(k)=\widetilde{C}_{i i} x_{i}(k) \text {. }
$$

From (11), the polytopic model of each control area is

$$
\begin{array}{r}
{\left[\widetilde{A}_{i i}(k) \widetilde{B}_{i i}(k) \cdots \widetilde{A}_{i j}(k) \widetilde{B}_{i j}(k) \cdots\right]} \\
=\sum_{\ell=1}^{L} \beta_{\ell}\left[\widetilde{A}_{i i}^{(\ell)} \widetilde{B}_{i i}^{(\ell)} \cdots \widetilde{A}_{i j}^{(\ell)} \widetilde{B}_{i j}^{(\ell)} \cdots\right] \in \Omega \\
\forall j \in\{1, \ldots, M\}, \quad j \neq i .
\end{array}
$$


TABLE 1: Variables and parameters used in thermal and hydro power plant.

\begin{tabular}{llc}
\hline Parameter/variable & Description & Unit \\
\hline$\Delta f(t)$ & Frequency deviation & Hz \\
$\Delta P_{g}(t)$ & Generator output power deviation & p.u. \\
$\Delta X_{g}(t)$ & Governor valve position deviation & p.u. \\
$\Delta P_{r}(t)$ & Reheater output deviation & p.u. \\
$\Delta X_{g h}(t)$ & Governor valve servomotor position deviation & p.u.MW \\
$\Delta P_{\text {tie }}(t)$ & Tie-line active power deviation & p.u.MW \\
$\Delta P_{d}(t)$ & Load disturbance & rad \\
$\Delta \delta(t)$ & Rotor angle deviation & Hz/p.u.MW \\
$K_{P}$ & Power system gain & p.u. \\
$K_{r}$ & Reheater gain & s \\
$T_{P}$ & Power system time constant & s \\
$T_{W}$ & Water starting time & s \\
$T_{1}, T_{2}, T_{R}$ & Hydrogovernor time constants & s \\
$T_{G}$ & Thermal governor time constant & s \\
$T_{T}$ & Turbine time constant & s \\
$T_{r}$ & Reheater time constant & p.u.MW \\
$T_{s}$ & Interconnection gain between CAs \\
$B$ & Frequency bias factor & p.u.MW/Hz \\
$R$ & Speed droop due to governor action & Hz/p.u.MW \\
ACE & Area control error & p.u.MW \\
\hline
\end{tabular}

$\widetilde{A}_{i i}(k), \widetilde{B}_{i i}(k), \widetilde{A}_{i j}(k), \widetilde{B}_{i j}(k)$, and $\widetilde{C}_{i i}$ are the relative matrices in the discrete-time model (11). $\Omega$ is the model parameter uncertainty set. $\beta_{\ell}$ 's are used to represent a convex combination of the model vertices since the convex hull (the polytope) is the extreme model vertices. Each vertex $\ell$ corresponds to a linear model. The states are assumed to be available.

\section{Robust Distributed Model Predictive Control Algorithm}

Considering the distributed discrete-time power system model (11), the min-max problem to be solved for each subsystem is expressed as

$$
\begin{aligned}
& \min _{u_{i}(k+l \mid k)} \max _{\left[\widetilde{A}_{i i}(k+l) \widetilde{B}_{i i}(k+l) \cdots \widetilde{A}_{i j}(k+l) \widetilde{B}_{i j}(k+l) \cdots\right], l \geq 0} J_{i}(k) \\
& \text { s.t. }\left|u_{i}(k+l \mid k)\right| \leq u_{i}^{\max }, \quad l \geq 0,
\end{aligned}
$$

where $J_{i}(k)$ is an object function for subsystem $i$ to guarantee the cooperation of subsystem controllers, defined as

$$
\begin{array}{r}
J_{i}(k)=\sum_{l=0}^{\infty}\left[x_{i}^{\prime}(k+l \mid k) S_{i} x_{i}(k+l \mid k)\right. \\
\left.\quad+u_{i}^{\prime}(k+l \mid k) R_{i} u_{i}(k+l \mid k)\right] \\
+\sum_{j \neq i}^{M} \sum_{l=0}^{\infty}\left[x_{j}^{\prime}(k+l \mid k) S_{j} x_{j}(k+l \mid k)\right. \\
\left.+u_{j}^{\prime}(k+l \mid k) R_{j} u_{j}(k+l \mid k)\right],
\end{array}
$$

where $x_{i}(k+l \mid k)$ and $u_{i}(k+l \mid k)$ are the predicted state and input variables for the $i$ th subsystem at time instant $k+l, l \geq 0$, based on data at time $k . S_{i}, R_{i}, S_{j}$, and $R_{j}$ are the weighting matrices.

The maximization is to choose time-varying model $\left[\widetilde{A}_{i i}(k+l) \widetilde{B}_{i i}(k+l) \cdots \widetilde{A}_{i j}(k+l) \widetilde{B}_{i j}(k+l) \cdots\right]$ in the uncertainty set $\Omega$ to get the worst situation of $J_{i}(k)$, and this worst situation will be minimized on the current and the future horizons.

To solve the optimal problem (13), it is necessary to find an upper bound of the object function (15). Considering the quadratic function

$$
V_{i}(\bar{x})=\bar{x}^{T} P_{i} \bar{x}, \quad P_{i}>0
$$

where $\bar{x}=\left[\begin{array}{llll}x_{1}^{\prime} & x_{2}^{\prime} & \cdots & x_{M}^{\prime}\end{array}\right]^{\prime}$. For all the subsystem $i, V_{i}(\bar{x})$ should satisfy the following stability constraint:

$$
\begin{aligned}
& V_{i}(\bar{x}(k+l+1 \mid k))-V_{i}(\bar{x}(k+l \mid k)) \\
& \leq-\left[x_{i}^{\prime}(k+l \mid k) S_{i} x_{i}(k+l \mid k)\right. \\
& +u_{i}^{\prime}(k+l \mid k) R_{i} u_{i}(k+l \mid k) \\
& +\sum_{j \neq i}^{M}\left(x_{j}^{\prime}(k+l \mid k) S_{j} x_{j}(k+l \mid k)\right. \\
& \left.\left.\quad+u_{j}^{\prime}(k+l \mid k) R_{j} u_{j}(k+l \mid k)\right)\right] .
\end{aligned}
$$

For $l=0,1, \ldots, \infty$, the accumulation of $(17)$ is

$$
V_{i}(\bar{x}(k \mid k)) \geq J_{i}(k) .
$$



be

So the upper bound of object function can be proved to

$$
\max _{\left[\widetilde{A}_{i i}(k+l) \widetilde{B}_{i i}(k+l) \cdots \widetilde{A}_{i j}(k+l) \widetilde{B}_{i j}(k+l) \cdots\right], l \geq 0} J_{i}(k) \leq V_{i}(\bar{x}(k \mid k)) .
$$

A state-feedback law is sought for each subsystem $i$ as follows:

$$
\begin{aligned}
u_{i}(k+l \mid k) & =f_{i i} x_{i}(k+l \mid k)+\sum_{j \neq i}^{M} f_{i j} x_{j}(k+l \mid k) \\
& =f_{i} \bar{x}(k+l \mid k),
\end{aligned}
$$

where $f_{i}=\left[\begin{array}{llll}f_{i 1} & f_{i 2} & \cdots & f_{i M}\end{array}\right]$.

When solving optimization problem of the subsystem $i$, the state-feedback law of the neighboring subsystem $j(j \neq i)$ is expressed as

$$
\begin{aligned}
u_{j}(k+l \mid k) & =f_{j j}^{*} x_{j}(k+l \mid k)+\sum_{j \neq s}^{M} f_{j s}^{*} x_{s}(k+l \mid k) \\
& =f_{j}^{*} \bar{x}(k+l \mid k),
\end{aligned}
$$

where $f_{j}^{*}=\left[\begin{array}{llll}f_{j 1}^{*} & f_{j 2}^{*} & \cdots & f_{j M}^{*}\end{array}\right]$.

The RDMPC algorithm will be redefined using statefeedback law (20) to minimize the upper bound

$$
\min _{u_{i}(k+l \mid k)} V_{i}(\bar{x}(k \mid k))=\min _{f_{i}} \bar{x}^{\prime}(k \mid k) P_{i} \bar{x}(k \mid k), \quad P_{i}>0 .
$$

For the whole power system, the expression of $\bar{x}$ is

$$
\begin{aligned}
& \bar{x}(k+1)=\left[\begin{array}{c}
x_{1}(k+1) \\
x_{2}(k+1) \\
\vdots \\
x_{M}(k+1)
\end{array}\right] \\
& =\left[\begin{array}{cccc}
\widetilde{A}_{11}(k) & \widetilde{A}_{12}(k) & \cdots & \widetilde{A}_{1 M}(k) \\
\widetilde{A}_{21}(k) & \widetilde{A}_{22}(k) & \cdots & \widetilde{A}_{2 M}(k) \\
\vdots & \vdots & & \vdots \\
\widetilde{A}_{M 1}(k) & \widetilde{A}_{M 2}(k) & \cdots & \widetilde{A}_{M M}(k)
\end{array}\right]\left[\begin{array}{c}
x_{1}(k) \\
x_{2}(k) \\
\vdots \\
x_{M}(k)
\end{array}\right] \\
& +\left[\begin{array}{c}
\widetilde{B}_{11}(k) \\
\widetilde{B}_{21}(k) \\
\vdots \\
\widetilde{B}_{M 1}(k)
\end{array}\right] u_{1}(k)+\left[\begin{array}{c}
\widetilde{B}_{12}(k) \\
\widetilde{B}_{22}(k) \\
\vdots \\
\widetilde{B}_{M 2}(k)
\end{array}\right] u_{2}(k) \\
& +\cdots+\left[\begin{array}{c}
\widetilde{B}_{1 M}(k) \\
\widetilde{B}_{2 M}(k) \\
\vdots \\
\widetilde{B}_{M M}(k)
\end{array}\right] u_{M}(k)
\end{aligned}
$$

Define

$$
\begin{gathered}
A(k)=\left[\begin{array}{cccc}
\widetilde{A}_{11}(k) & \widetilde{A}_{12}(k) & \cdots & \widetilde{A}_{1 M}(k) \\
\widetilde{A}_{21}(k) & \widetilde{A}_{22}(k) & \cdots & \widetilde{A}_{2 M}(k) \\
\vdots & \vdots & & \vdots \\
\widetilde{A}_{M 1}(k) & \widetilde{A}_{M 2}(k) & \cdots & \widetilde{A}_{M M}(k)
\end{array}\right], \\
B_{i}(k)=\left[\begin{array}{c}
\widetilde{B}_{1 i}(k) \\
\widetilde{B}_{2 i}(k) \\
\vdots \\
\widetilde{B}_{M i}(k)
\end{array}\right] .
\end{gathered}
$$

Using (20) and (21), the state (23) can be simplified as

$$
\bar{x}(k+1)=\left[\bar{A}(k)+B_{i}(k) f_{i}\right] \bar{x}(k)
$$

in which $\bar{A}(k)=A(k)+\sum_{j \neq i}^{M} B_{j}(k) f_{j}^{*}$.

The robust stability constraint in (17) becomes

$$
\begin{aligned}
& {\left[\bar{A}^{(\ell)}(k+l)+B_{i}^{(\ell)}(k+l) f_{i}\right]^{\prime}} \\
& \quad \times P_{i}\left[\bar{A}^{(\ell)}(k+l)+B_{i}^{(\ell)}(k+l) f_{i}\right]-P_{i} \\
& \leq-\left(\bar{S}_{i}+\sum_{j \neq i}^{M} f_{j}^{* \prime} R_{j} f_{j}^{*}+f_{i}^{\prime} R_{i} f_{i}\right),
\end{aligned}
$$

where

$$
\bar{S}_{i}=\left[\begin{array}{llll}
S_{1} & & & \\
& S_{2} & & \\
& & \ddots & \\
& & & S_{M}
\end{array}\right] .
$$

By defining an upper bound,

$$
J_{i}(k) \leq V_{i}(\bar{x}(k \mid k)) \leq \gamma_{i}
$$

The optimal problem (22) is equivalent to

$$
\begin{array}{ll}
\min _{\gamma_{i}, P_{i}} & \gamma_{i} \\
\text { s.t. } & \bar{x}^{\prime}(k \mid k) P_{i} \bar{x}(k \mid k) \leq \gamma_{i} .
\end{array}
$$

Substituting $P_{i}=\gamma_{i} Q_{i}^{-1}>0, Y_{i}=f_{i} Q_{i}$, with the input constraints given in (13) and the stability constraint (27), followed by a Schur complement decomposition, the minimization of $J_{i}(k)$ can be replaced by the minimization 
problem (30) as in the following linear minimization problem with LMI constraints:

$$
\begin{gathered}
\min _{\gamma_{i}, P_{i}, Q_{i}} \gamma_{i} \\
\text { s.t. } \quad\left[\begin{array}{ccc}
1 & \bar{x}^{\prime}(k \mid k) \\
\bar{x}(k \mid k) & Q_{i}
\end{array}\right] \geq 0, \\
{\left[\begin{array}{cccc}
Q_{i} & Q_{i} \bar{A}^{\prime(\ell)}+Y_{i}^{\prime} B_{i}^{\prime(\ell)} & Q_{i} \bar{S}_{i}^{1 / 2} & Y_{i} R_{i}^{1 / 2} \\
\bar{A}^{(\ell)} Q_{i}+B_{i}^{(\ell)} Y_{i} & Q_{i} & 0 & 0 \\
\bar{S}_{i}^{1 / 2} Q_{i} & 0 & \gamma_{i} I & 0 \\
R_{i}^{1 / 2} Y_{i} & 0 & 0 & \gamma_{i} I
\end{array}\right] \geq 0} \\
\\
\left.\quad \begin{array}{cc}
\left(u_{i}^{\max }\right)^{2} I & Y_{i} \\
Y_{i}^{\prime} & Q_{i}
\end{array}\right] \geq 0 .
\end{gathered}
$$

For the constraints on power system state

$$
\max _{\left[\widetilde{A}_{i i}(k+l) \widetilde{B}_{i i}(k+l) \cdots \widetilde{A}_{i j}(k+l) \widetilde{B}_{i j}(k+l) \cdots\right] \in \Omega, l \geq 0}\left\|y_{i}(k+l \mid k)\right\|_{2} \leq y_{i, \max } .
$$

Transform it to LMI form as

$$
\begin{aligned}
& {\left[\begin{array}{cc}
Q_{i} & \left(\bar{A}^{(\ell)} Q_{i}+B_{i}^{(\ell)} Y_{i}\right)^{\prime} \widetilde{C}_{i i}^{\prime} \\
\widetilde{C}_{i i}\left(\bar{A}^{(\ell)} Q_{i}+B_{i}^{(\ell)} Y_{i}\right) & y_{i, \max }^{2} I
\end{array}\right] \geq 0} \\
& \ell=1,2, \ldots, L \text {. }
\end{aligned}
$$

\section{The Simulation}

Two examples are considered to demonstrate the effectiveness of the proposed RDMPC. In the first one, the RDMPC is utilized in a two-control area thermal power system, while in the second one, a three-area thermal-hydro power system is considered.

Case 1 (a two-control area thermal power system). A twocontrol area thermal power system is shown in Figure 3. The parameters used in the simulation is as follows:

$$
\begin{gathered}
K_{P 1}=120 \mathrm{~Hz} / \text { p.u.MW } \quad K_{P 2}=75 \mathrm{~Hz} / \text { p.u.MW; } \\
T_{P 1}=20 \mathrm{~s} ; \quad T_{P 2}=15 \mathrm{~s} ; \quad K_{r 1}=K_{r 2}=0.5 \mathrm{~Hz} / \text { p.u.MW; } \\
R_{1}=2.4 \mathrm{~Hz} / \text { p.u.MW; } \quad R_{2}=3 \mathrm{~Hz} / \text { p.u.MW; } \\
B_{1}=0.425 \text { p.u.MW } / \mathrm{Hz} ; \quad B_{2}=0.347 \text { p.u.MW } / \mathrm{Hz} ; \\
T_{G 1}=0.08 \text { s; } \quad T_{G 2}=0.2 \mathrm{~s} ; \quad T_{T 1}=T_{T 2}=0.3 \mathrm{~s} ; \\
T_{s 12}=0.545 \text { p.u.MW; } \quad T_{r 1}=T_{r 2}=10 \mathrm{~s} .
\end{gathered}
$$

The power system model in Figure 1 with included GRC is shown in Figure 4. In simulations, GRC was set to $\left|\Delta \dot{P}_{g i}\right| \leq$ $r=0.0017$ p.u.MW/s.

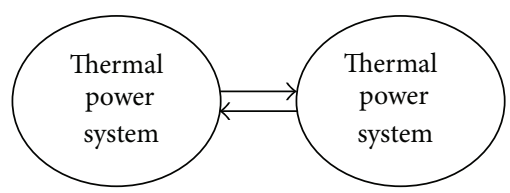

FIgURE 3: The two-control area interconnected thermal power system.

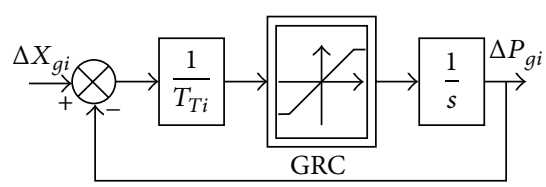

Figure 4: GRC in power system LFC.

In real time power system LFC, the power system time constant $T_{P}$ and turbine time constant $T_{T}$ can change frequently. Thus the robustness study is performed by applying intentional changes in these two parameters. The maximum range of parameter variation is chosen to be $40 \%$. The polytopic of uncertain LFC system has four vertices, which are

$$
\begin{array}{ll}
A_{i}^{(1)}\left(0.6 T_{P i}, 0.6 T_{T i}\right) ; & A_{i}^{(2)}\left(1.4 T_{P i}, 1.4 T_{T i}\right) ; \\
A_{i}^{(3)}\left(0.6 T_{P i}, 1.4 T_{T i}\right) ; & A_{i}^{(4)}\left(1.4 T_{P i}, 0.6 T_{T i}\right) .
\end{array}
$$

Under the parameter changes, the performance of the RDMPC is assessed by applying load disturbance. At $t=2 \mathrm{~s}$, a step load disturbance on control area is added to be $\Delta P_{d 1}=$ 0.01 p.u. Choose the sample time to be $T_{s}=0.1 \mathrm{~s}, S_{i}=1$, and $R_{i}=0.05$.

The proposed RDMPC is compared with two other schemes, for example, the conventional robust centralized MPC, which solves the min-max optimization problem using the centralized model by the formulation of a linear matrix inequality and also with the communicated-based MPC, which utilizes the objective function for local subsystem only. Figures 5, 6, and 7 show the comparison results of the ACE signals, the frequency deviations, and the tie-line power flow, respectively. It is clear that the proposed RDMPC has the best performance, since the MPC controllers cooperate with each other in achieving system-wide objectives. The performance of the robust centralized MPC is quite close to that of the $\mathrm{RDMPC}$, since it is also robust to parameter changes. The only shortcoming of the centralized MPC is its high computation burden. The performance of the communicated-based MPC is the worst, since it can neither realize the cooperation of the subsystems nor adapt to parameter changes.

Case 2 (a three-area thermal-hydro power system). The three-control area interconnected power system containing thermal and hydro power plant is showed in Figure 8.

The power system model in Figures 1 and 2 with included GRC in hydro power plant is shown in Figure 9, where $\left|\Delta \dot{P}_{g i}\right| \leq r=0.045$ p.u.MW/s. 


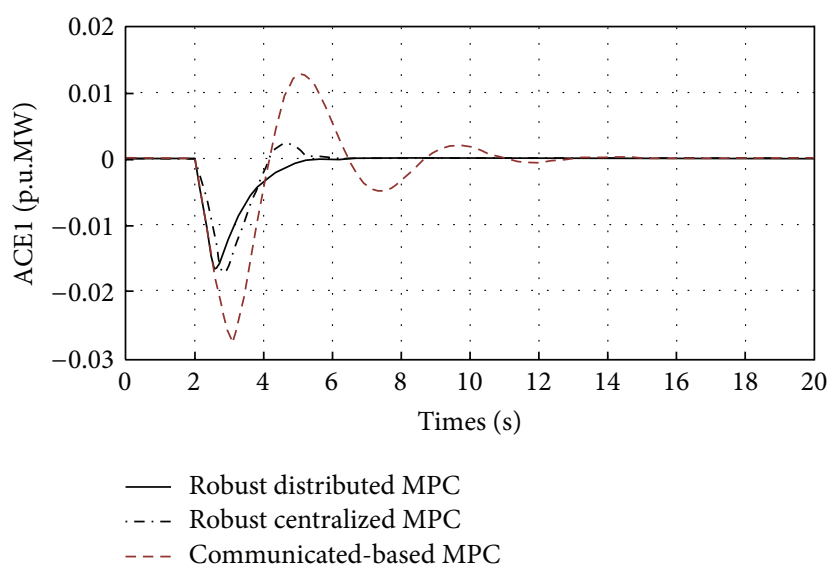

(a)

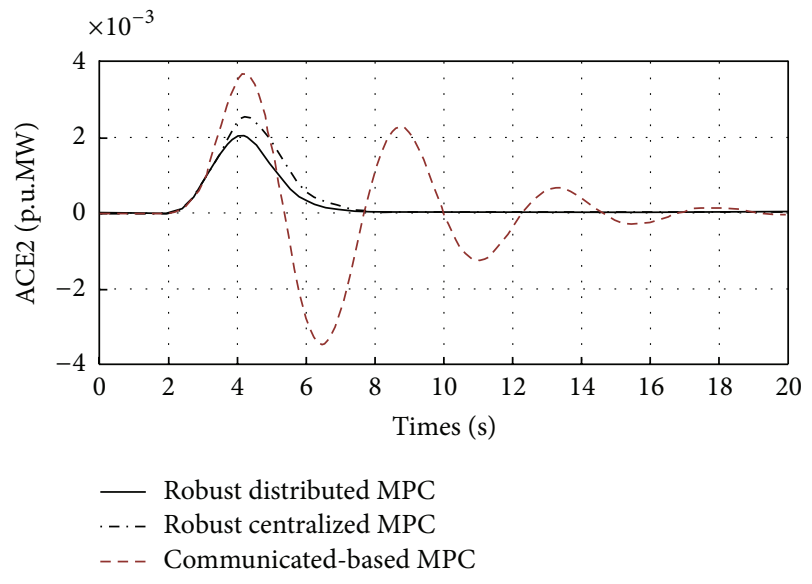

(b)

Figure 5: ACE signals in the two subsystems.

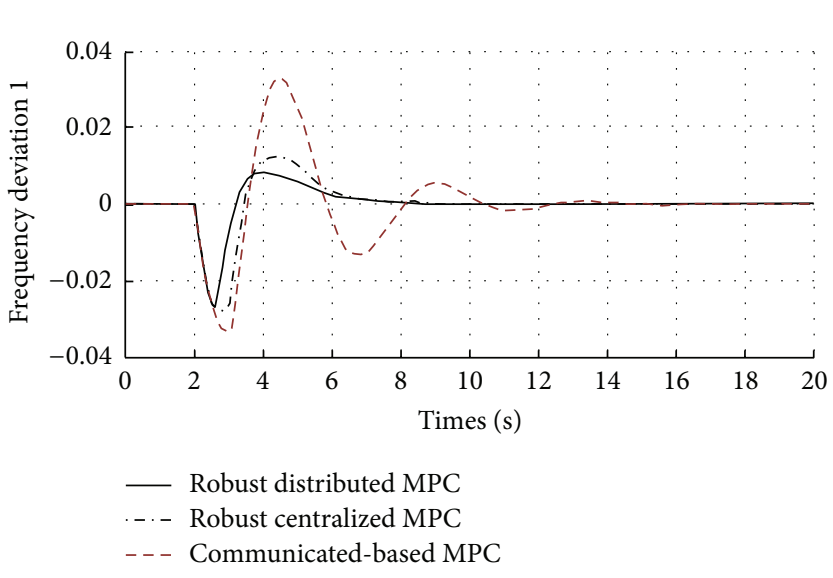

(a)

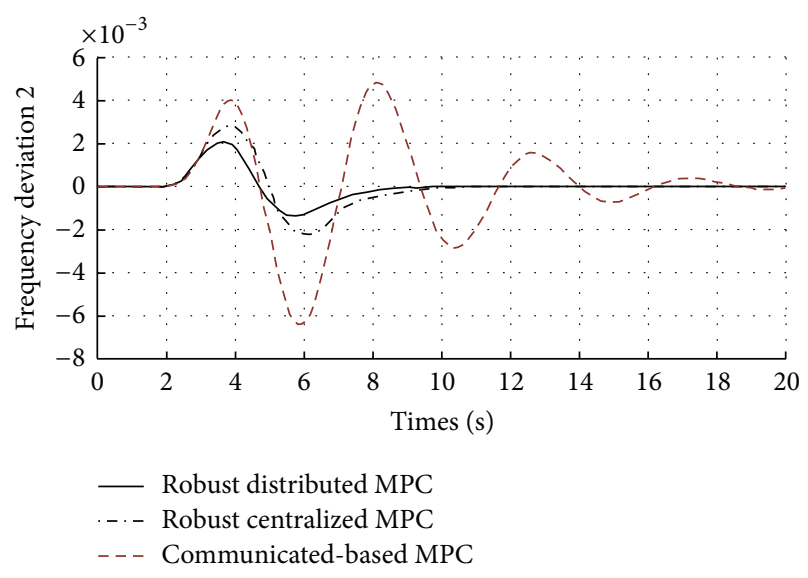

(b)

Figure 6: The frequency deviations.

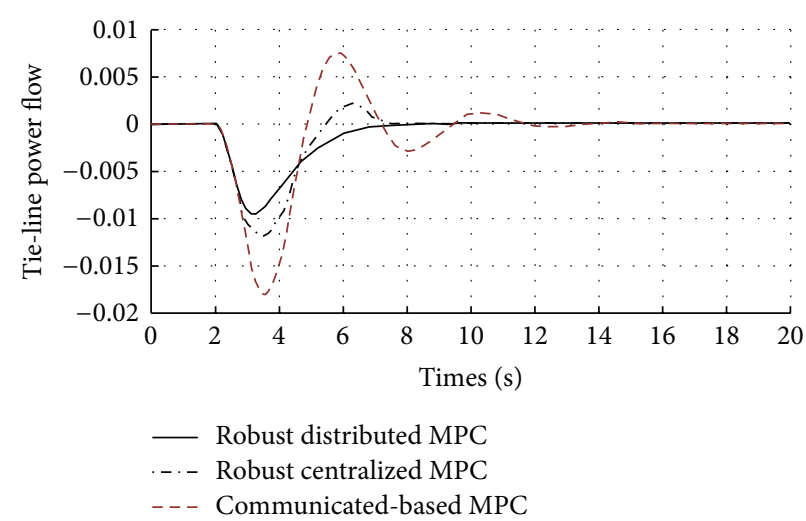

FIgURE 7: The tie-line power flow between two control areas. 


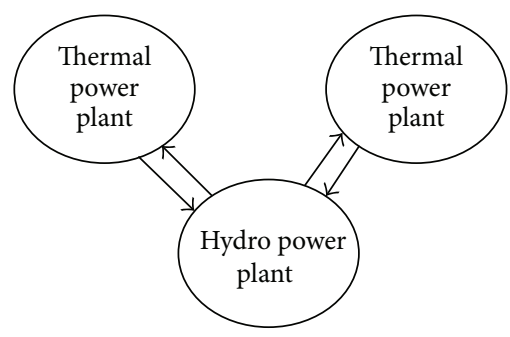

FIGURE 8: The three-control area interconnected thermal-hydro power system.

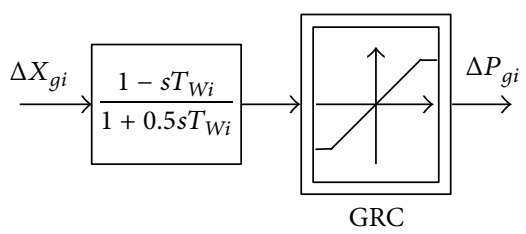

FIGURE 9: GRC in hydro power system.
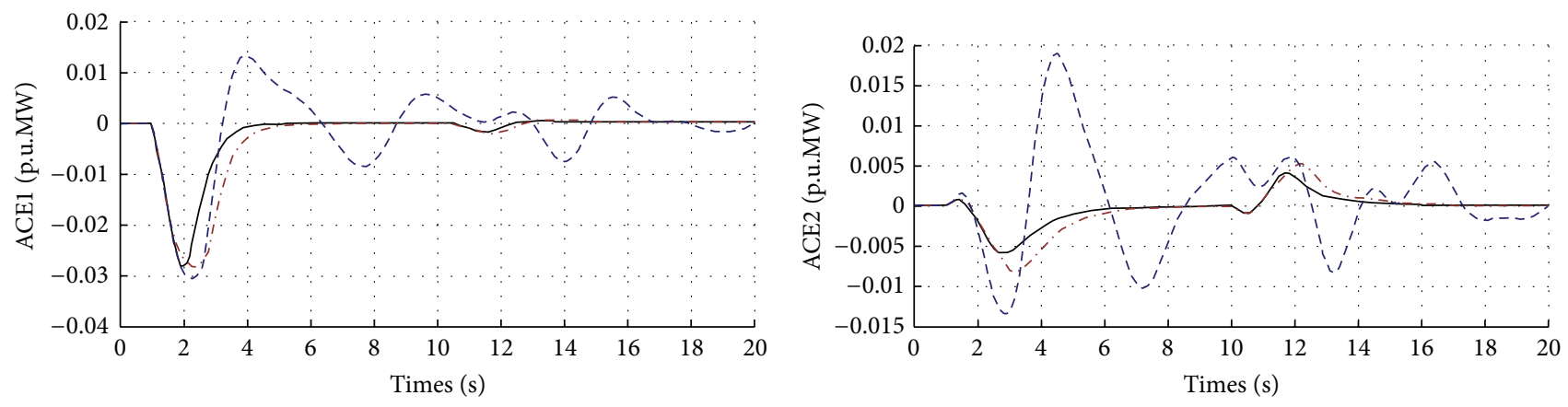

- Robust distributed MPC

... - Robust centralized MPC

— Robust distributed MPC

-. Robust centralized MPC

- - - Communicated-based MPC

- - - Communicated-based MPC

(a)

(b)

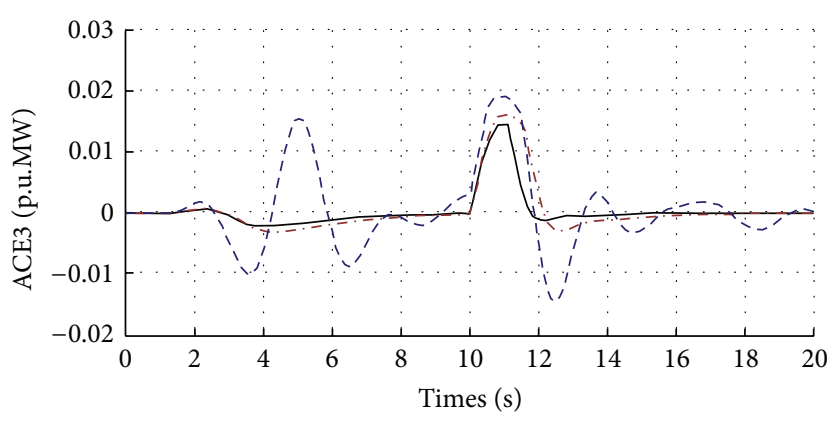

— Robust distributed MPC

...- Robust centralized MPC

- - - Communicated-based MPC

(c)

FIGURE 10: ACE signals in three-area thermal-hydro power system. 


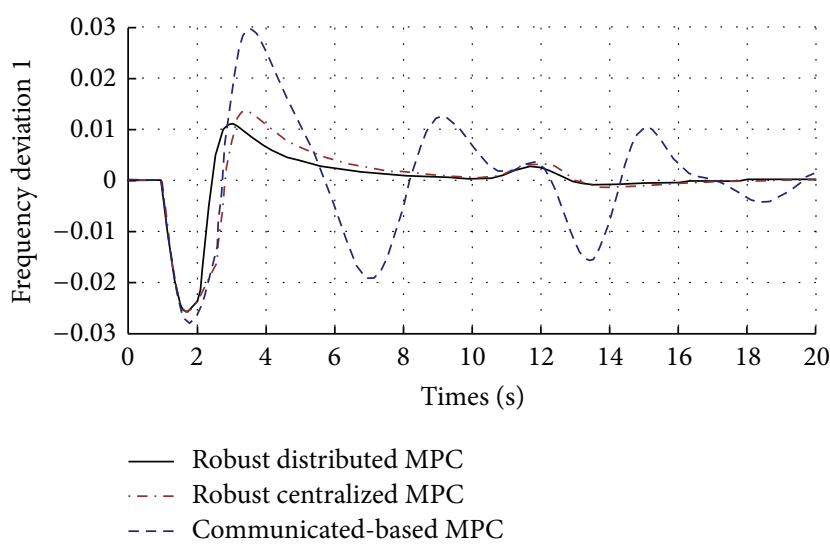

(a)

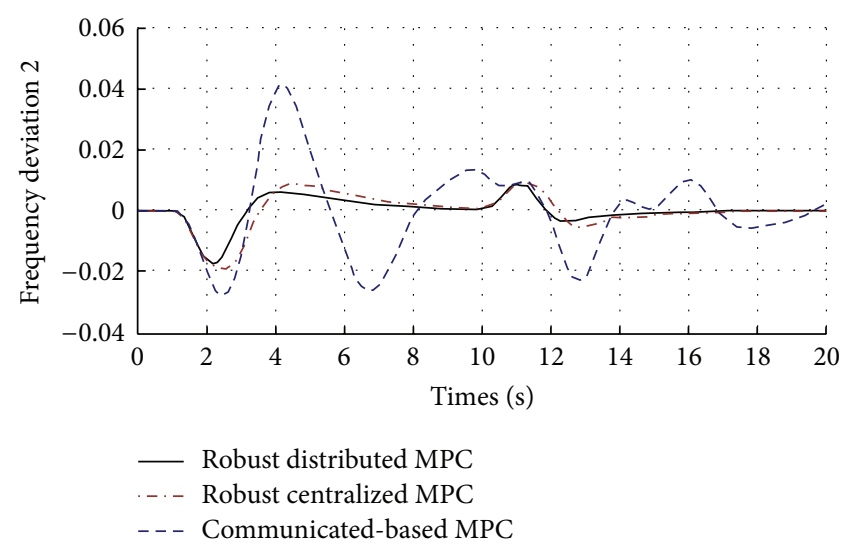

(b)

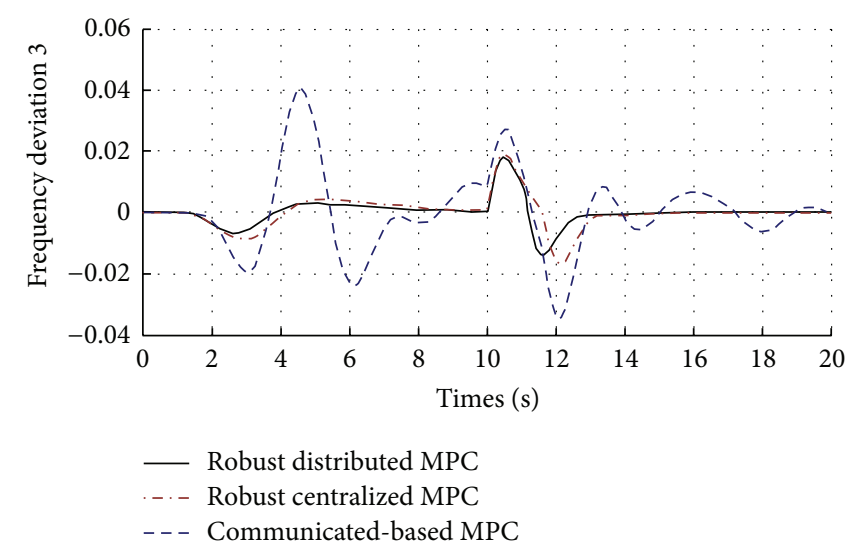

(c)

FIgURE 11: The frequency deviation in the three-area thermal-hydro power system.

The parameters used in the simulation are as follows:

$$
\begin{gathered}
K_{P 1}=120 \mathrm{~Hz} / \text { p.u.MW; } \quad K_{P 3}=115 \mathrm{~Hz} / \text { p.u.MW; } \\
K_{P 3}=75 \mathrm{~Hz} / \text { p.u.MW; } \quad T_{P 1}=20 \mathrm{~s} ; \quad T_{P 2}=20 \mathrm{~s} ; \\
T_{P 3}=15 \mathrm{~s} ; \quad K_{r 1}=K_{r 3}=0.5 \mathrm{~Hz} / \text { p.u.MW; } \\
R_{1}=2.4 \mathrm{~Hz} / \text { p.u.MW; } \quad R_{2}=2.5 \mathrm{~Hz} / \text { p.u.MW; } \\
R_{3}=3 \mathrm{~Hz} / \text { p.u.MW; } \quad B_{1}=0.425 \text { p.u.MW } / \mathrm{Hz} ; \\
B_{2}=0.494 \text { p.u.MW } / \mathrm{Hz} ; \quad B_{3}=0.347 \text { p.u.MW } / \mathrm{Hz} ; \\
T_{R 2}=0.6 \mathrm{~s} ; \quad T_{1(2)}=48.7 \mathrm{~s} ; \quad T_{2(2)}=5 \mathrm{~s} ; \\
T_{W 2}=1 \mathrm{~s} ; \quad T_{G 1}=0.08 \mathrm{~s} ; \quad T_{G 3}=0.2 \mathrm{~s} ; \\
T_{T 1}=T_{T 3}=0.3 \mathrm{~s} ; \quad T_{r 1}=T_{r 3}=10 \mathrm{~s} ; \\
T_{s 12}=0.545 \text { p.u.MW; } \quad T_{s 23}=0.545 \text { p.u.MW } .
\end{gathered}
$$

Since the maximum range of parameter variation is also chosen to be $40 \%$ for hydro power system, the polytope is

$$
A_{2}^{(1)}\left(0.6 T_{P 2}\right) ; \quad A_{2}^{(2)}\left(1.4 T_{P 2}\right) .
$$

At $t=1 \mathrm{~s}$, a step load disturbance on control area 1 is added as $\Delta P_{d 1}=0.01$ p.u., and at $t=10 \mathrm{~s}$, a step load disturbance on control area 3 is added as $\Delta P_{d 3}=-0.01$ p.u. Figures 10 and 11 show the comparison results of the ACE signals and the frequency deviations, demonstrating clearly the advantage of the proposed RDMPC.

\section{Conclusion}

In this paper, a robust distributed MPC scheme for load frequency control of interconnected power system is presented. The overall system consisted of at least two control areas, which either can be thermal-thermal power system or thermal-hydro power system. Each control area has its own polytopic distributed model in order to consider the uncertainty because of parameter variation. A min-max cost function is used for the optimization problem, and the LMI method is involved to solve this problem. The simulation results illustrate the advantage of the proposed RDMPC, due to its cooperative function. Thus it is suitable for LFC of power system, which is large-scale complex system and subject to parameter uncertainty. 


\section{Conflict of Interests}

The authors declare that there is no conflict of interests regarding the publication of this paper.

\section{Acknowledgments}

This work was supported by National Natural Science Foundation of China under Grants 60974051, 61273144, and 61203041, Natural Science Foundation of Beijing under Grant 4122071, Chinese National Postdoctoral Science Foundation under Grants 2011M500217 and 2012T50036, and the Doctoral Fund of Ministry of Education of China under Grant 20120036120013.

\section{References}

[1] R. K. Cavin, M. C. Budge, and P. Rasmussen, "An optimal linear system approach to load-frequency control," IEEE Transactions on Power Apparatus and Systems, vol. 90, no. 6, pp. 2472-2482, 1971.

[2] S. Yin, H. Luo, and S. Ding, "Real-time implementation of fault tolerant control system with performance optimization," IEEE Transactions on Industrial Electronics, vol. 61, no. 5, pp. 24022411, 2013.

[3] S. Ding, S. Yin, K. Peng, H. Hao, and B. Shen, "A novel scheme for key performance indicator prediction and diagnosis with application to an industrial hot strip mill," IEEE Transactions on Industrial Informatics, vol. 9, no. 4, pp. 2239-2247, 2012.

[4] T. Li, W. X. Zheng, and C. Lin, "Delay-slope-dependent stability results of recurrent neural networks," IEEE Transactions on Neural Networks, vol. 22, no. 12, pp. 2138-2143, 2011.

[5] Y. Wang, R. Zhou, and C. Wen, "Robust load-frequency controller design for power systems," IEE Proceedings C, vol. 140, no. 1, pp. 11-16, 1993.

[6] A. M. Stanković, G. Tadmor, and T. A. Sakharuk, "On robust control analysis and design for load frequency regulation," IEEE Transactions on Power Systems, vol. 13, no. 2, pp. 449-455, 1998.

[7] X. Yu and K. Tomsovic, "Application of linear matrix inequalities for load frequency control with communication delays," IEEE Transactions on Power Systems, vol. 19, no. 3, pp. 1508-1515, 2004.

[8] H. J. Lee, J. B. Park, and Y. H. Joo, "Robust load-frequency control for uncertain nonlinear power systems: a fuzzy logic approach," Information Sciences, vol. 176, no. 23, pp. 3520-3537, 2006.

[9] H. Shayeghi, H. A. Shayanfar, and O. P. Malik, "Robust decentralized neural networks based LFC in a deregulated power system," Electric Power Systems Research, vol. 77, no. 3-4, pp. 241-251, 2007.

[10] D. Rerkpreedapong, A. Hasanović, and A. Feliachi, "Robust load frequency control using genetic algorithms and linear matrix inequalities," IEEE Transactions on Power Systems, vol. 18, no. 2, pp. 855-861, 2003.

[11] M. V. Kothare, V. Balakrishnan, and M. Morari, "Robust constrained model predictive control using linear matrix inequalities," Automatica, vol. 32, no. 10, pp. 1361-1379, 1996.

[12] X. Liu, S. Feng, and M. Ma, "Robust MPC for the constrained system with polytopic uncertainty," International Journal of Systems Science, vol. 43, no. 2, pp. 248-258, 2012.
[13] A. N. Venkat, I. A. Hiskens, J. B. Rawlings, and S. J. Wright, "Distributed MPC strategies with application to power system automatic generation control," IEEE Transactions on Control Systems Technology, vol. 16, no. 6, pp. 1192-1206, 2008.

[14] T. H. Mohamed, H. Bevrani, A. A. Hassan, and T. Hiyama, "Decentralized model predictive based load frequency control in an interconnected power system," Energy Conversion and Management, vol. 52, no. 2, pp. 1208-1214, 2011.

[15] W. Al-Gherwi, H. Budman, and A. Elkamel, "A robust distributed model predictive control algorithm," Journal of Process Control, vol. 21, no. 8, pp. 1127-1137, 2011.

[16] K. Vrdoljak, N. Perić, and I. Petrović, "Sliding mode based loadfrequency control in power systems," Electric Power Systems Research, vol. 80, no. 5, pp. 514-527, 2010. 


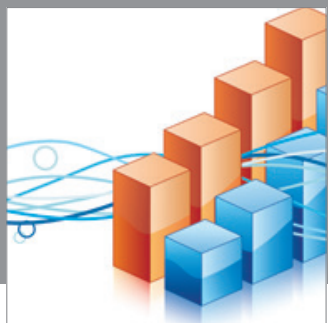

Advances in

Operations Research

mansans

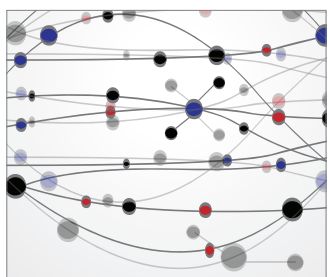

The Scientific World Journal
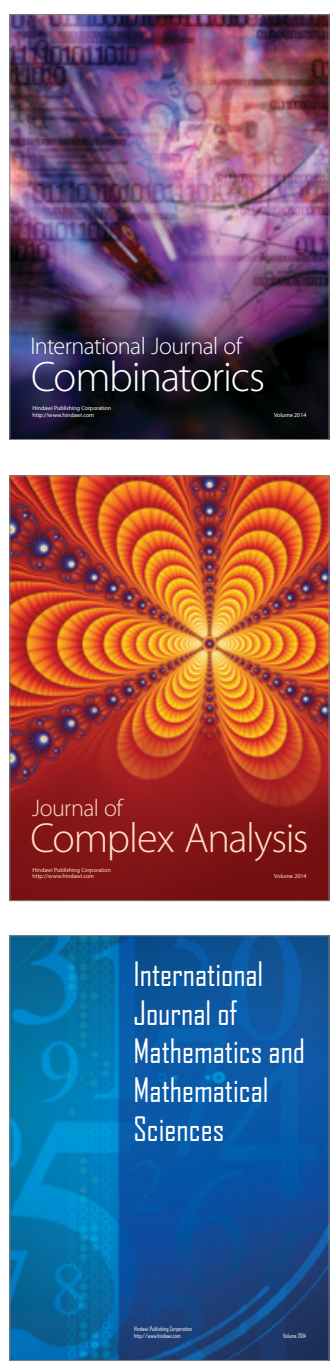
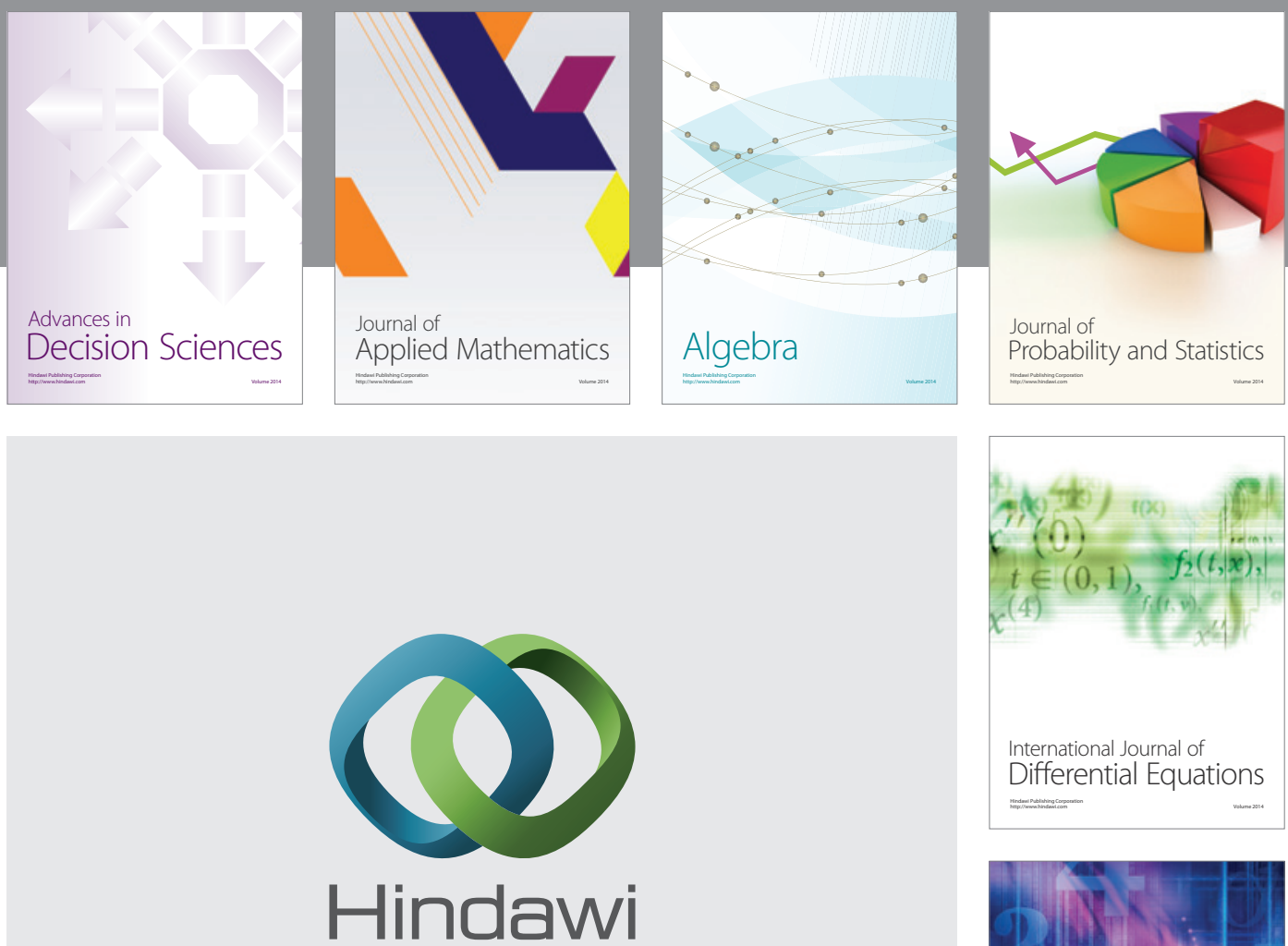

Submit your manuscripts at http://www.hindawi.com
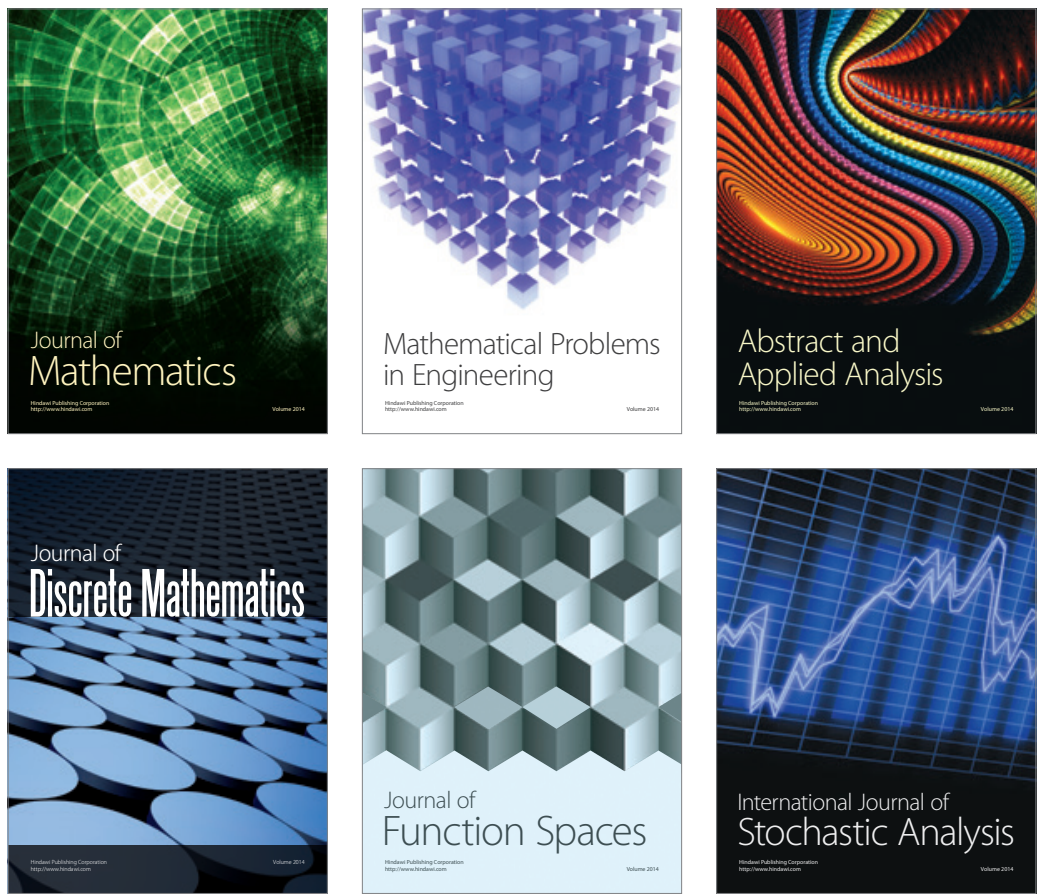

Journal of

Function Spaces

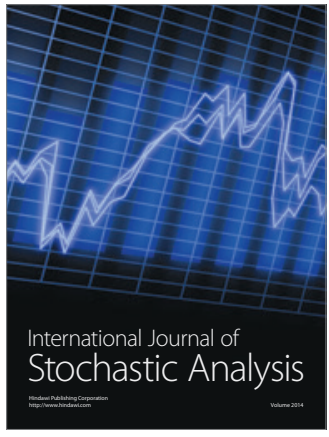

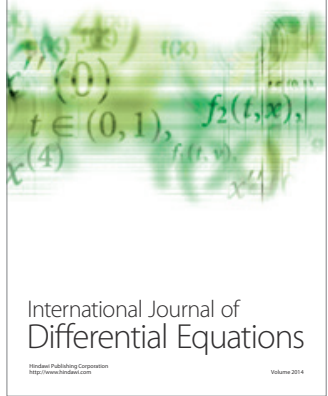
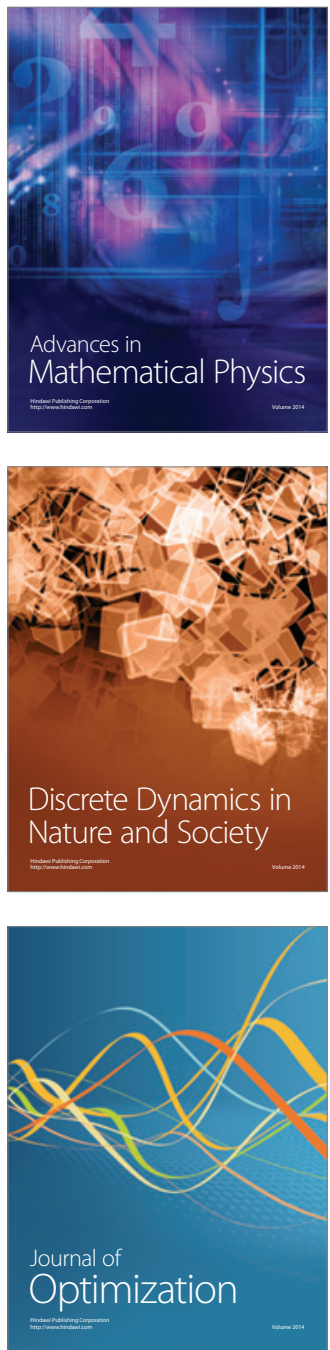